\title{
Encounters with Masters
}

\section{by Lizhen Ji}

\author{
University of Michigan
}

From January 4 to January 8, 2013, the inaugural Master Lectures on Mathematics were held in Sanya.

The following five masters in mathematics gave plenary talks:

- David Mumford (Brown University), The partnership of pure and applied mathematics

- Richard Schoen (Stanford University), Minimal submanifolds in differential geometry

- Wilfried Schmid (Harvard University), Harmonic analysis on Lie groups

- Stanislar Smirnov (University of Genava), Discrete complex analysis and probability

- Shing-Tung Yau (Harvard University), Geometry: from Riemann to Einstein and onto String Theory

These five mathematicians are true masters in every sense of the word. They are deeply concerned with both research and education in mathematics. Three of them hold the illustrious Fields medal.

In order to follow the theme of learning from masters, as well as to encourage the students at the conference, a panel discussion titled "Encounter with Masters" was organized on the evening of January 7 . The following is the transcript of the conversations from that night.

\section{Question 1}

Lizhen Ji: In the most famous book in the world, one passage says: ask and you will be given. So I think I will start by asking our masters and you guys will ask more. As we all know, examples are important in mathematics and also in daily life. Since examples are important, let me ask the masters here to give us one example in their lives which has played a very special role in either their lives or in mathematics. Let me start with David Mumford.

Mumford: One thing which made me fall in love with algebraic geometry was taking a course on algebraic curves and doing a paper about blowing up the projective plane to desingularize singularities of plane curves. Take for example $y^{2}=x^{3}$, the simplest singular curve. What you do is to take the singular point, and blowing up replaces the point by the whole curve of directions through that point, called the exceptional locus. And then the singularity, all of a sudden, is changed into a simpler singularity. This idea, that you take the space and con- tinually transform it by this remarkable algebraic process, it just seemed so exotic and fascinating, and so I fell in love with algebraic geometry.

Schmid: Well, I'm not quite sure what I should say here. I suppose that one thing was important for me in becoming a mathematician was an experience I had as a high school student in Germany. High school in Germany started at age 10. My father was professor of Latin, and so I went to a school that emphasized Latin, but I was more interested in mathematics. Around the time I was 14, I complained to my parents that my school did not teach enough mathematics. One of our neighbors was Friedrich Hirzebruch, then the most influential mathematician in Germany. My father talked to him, and he said, well, that's not a problem, I will give your son private lessons. So when I was 14, I got private lessons from the best known mathematician in Germany at that time.

Smirnov: What made me choose mathematics? Well, it happened gradually, so it is hard to pinpoint one reason. I grew up with my grandparents, and my grandfather graduated from the math department. But then the Second World War started and he became an engineer-even getting a second education in engineering. All his life he worked between applied mathematics and engineering, and, somehow, when I was young I was imagining myself an engineer, building airplanes or spaceships, rather than a mathematician. I think one reason was the impression that I received in school, that mathematics is a science done by the ancients, and that it was finished a hundred years ago and there would be no new theorems. Even when learning physics and biology in school problems they mentioned some modern developments. All that changed when I realized that this was not the case, maybe after some university lectures I attended while in high school. I gradually became attracted by mathematics.

In regards to choosing areas, I think now is actually as exciting a time for mathematics as ever. It is certainly very different from a hundred, or even forty, years ago in the sense that now to arrive at interesting things in most areas, you have to learn much more. But it is not all that out of reach-there are many open problems that one can start thinking about, even at the undergraduate level. And also what is interesting nowadays is the interaction between different areas of mathematics, as well as with other sciences-physics, biology, economics, etc.-is on the rise. I find this very exciting. 
Shing-Tung Yau: I started to be interested in mathematics only after I studied geometry in middle school. In my second year in middle school, I learned the axioms of plane geometry. I was surprised by the elegance of mathematics and started to fall in love with it. I pursued the subject of functional analysis for quite a while when I was in college. My view of mathematics changed when I arrived at Berkeley for my graduate study, where I learned differential geometry and differential equations. While I was majorly interested in geometry, I found that many complicated problems in geometry can be phrased in terms of nonlinear partial differential equations, while, at the same time, when these equations are interpreted correctly in terms of geometry, they can be solved much more easily. The power of analysis in geometry excited me.

\section{Question 2}

Lizhen Ji: Okay, so I think that besides having masters from around the world, I believe we have the best students coming from China. So we are expecting the best questions from the audience.

Student: My questions are for all of the masters. My first question is: what is the most unforgettable difficulty in your studies or research? Secondly: how did you get through that hard time?

Mumford: What happened to me is that, half a dozen times, I thought I had proven $\mathrm{X}$ and not $\mathrm{X}$ both. You ask, "what the hell is happening?" But then you say, "now it is time to really figure something out. Time to find out how I could have been so stupid." This will help me a lot-it is total confusion until you get some clear ideas and get it straight after all.

Smirnov: If we find a contradiction in mathematics, we will all get fired.

Mumford: Yeah, that is also possible. (laugh)

Schmid: Yes, I certainly had experience of this sort-every mathematician does-but I want to talk about a different kind of difficulty. When you are a mathematician and think about important questions to study, there will be periods when somehow ideas don't seem to flow. That happens to most of us, maybe not to everyone, and maybe some of us are luckier. But I have certainly been in the situations where I found myself frustrated because no ideas came, and nothing seemed to work. When that happens, then you have to persist, and you have to keep working. You must be able to tell yourself that you will eventually find a solution and you will get over the difficulty.

Smirnov: I have been in this situation too, often for months, even for years. Sometimes it's clear what the theorem should be, and you have ideas on how to approach it, but you get stuck trying to do one of the steps.
If you persist, hopefully it will eventually come up. There are many examples when persistence helped after many years. Lennart Carleson proved the famous theorem, about almost everywhere convergence of Fourier series for square integrable functions, after nine years. Everyone-Zygmund, Kolmogorov, and soon-thought there should be a counterexample, and Lennart spent years trying to construct the ultimate one. But it didn't work, and he understood that perhaps it means that there is no counterexample at all, and he proved the theorem. So, one should work hard. But if you are stuck, you probably shouldn't concentrate on only one problem. You should take breaks. Richard Feynman, a famous physicist who was also very good at mathematics, used to say that he liked to teach because when in research you get stuck and depressed, then you got to work with the students.

Shing-Tung Yau: I found it challenge when interesting questions are being studied. There are always difficulties to overcome. But fresh ideas can be gained by working with friends from different subjects. In the past forty years, I have been working on subjects relating mathematics to theoretical physics, and I enjoyed it tremendously. But it is not an easy thing to do because I was not trained as a physicist. I need to learn from physicists. The subjects that I started with were general relativity and later string theory, especially questions related to mirror symmetry. It was not easy to understand what the physicists wanted to say at the beginning, and I knew little about the subject. But many of my good friends were doing research in the same topics; hence I can learn more quickly. I think one can do research on a subject that you may not know well. One can also learn faster if there are good friends to help. In many cases, I was shocked by the intuition that other subjects can provide for mathematics. Some of the ideas were totally unexpected. For example, when my postdoctoral fellow Brian Greene told me about the idea of mirror symmetry for Calabi-Yau manifolds, I was surprised. Even though it may be difficult, and even challenging, to get into a field that one does not really know well, one can often find ideas from other subjects to solve problems that one wanted to solve for a long time. Fortunately, I have many good friends. After working with them, I could understand their subjects much better. Of course, I also provided ideas that can help them. Good communication is important. Over all, to cross into a new field is not easy. But if one works hard that can be overcome, especially when you are young.

\section{Question 3}

Pengyu Le: When you were a student, who was the mathematician you admired most? What did you learn from him or her?

Lizhen Ji: In other words, your mathematics hero and his or her impact on you.

Mumford: For me there is one person, without any 
doubt, who doesn't seem to come from the planet earth. That was Alexander Grothendieck. He is truly a founder of new ideas. I have never met or read anybody who could take a question, formulate the most abstract point of view on it and somehow gain some deep insight from this.

Schmid: It is not as clear for me-there is no obvious answer. Certainly, one person, one mathematician, that I admire is Michael Atiyah. In my early thirties, I met Atiyah at the Institute for Advanced Study. He was interested in a problem that I was interested in too. So, we worked on this problem together. In that collaboration, I think, I learned more than I had learned from classes when I was a student. One can learn from one's collaborators, and sometimes one can learn a great deal.

Smirnov: I think for me, besides my advisors, people who influenced me most are Dennis Sullivan and Lennart Carleson. With Dennis, I accidentally met him when I was an undergraduate working on my masters thesis, and he, by correspondence, helped me with a problem in dynamical systems. What is interesting is that my result, in the end, answered a question he was interested in. Since then I have visited him many times and we collaborated. Dennis has tremendous intuition and he is very generous with his colleagues and students; he always wants to share his ideas. And a few times in his life, he proposed a very simple answer to dramatically complex questions-you learn one idea, and suddenly the whole setup becomes clear.

I met Lennart Carleson when I was a graduate student at Caltech, and then he invited me to Stockholm. I had read most of his papers before, as he was a towering figure in several areas of analysis, and I never expected I would meet him, let alone work together. I already mentioned his persistence and willingness to push really hard and work out difficult places which seemed beyond possible. I also admire Lennart's willingness to completely change his area, which he did several times in his life. It requires quite some courage to start from scratch, and from learn basic things. It is always a gamble, but can be very rewarding.

Shing-Tung Yau: I do not have a single hero. I have been influenced by a large group of mathematicians. Among the senior ones there are Chern, Calabi, Milnor, Morrey, Nirenberg, Pogorelov, and Singer. They are inspiring and they exposed me to many directions in mathematics that I had not tried before. But my experience with them was different from working with my younger coauthors, from which I learned down-to-earth technology and the means to do calculations. The most important coauthors for me in the seventies and eighties were S. Y. Cheng, Richard Schoen, Leon Simon, Karen Uhlenbeck, and Peter Li, among others. Cheng worked with me on differential equations over manifolds, on real and complex Monge-Ampère equations, on maximal hypersurfaces, and affine spheres. We developed some new technology for geometric analysis; Schoen and Simon worked with me on minimal submanifolds, harmonic maps, general relativity, and problems in topology; Karen Uhlenbeck on Hermitian Yang Mills connections; Peter Li on eigenfunctions and the heat equations producing the fundamental inequality of the heat equation. I am most grateful to the inspiring experiences I had while working with them. Not only for the elegant results we produced, but also the interesting technology we developed.

During the nineties and up until now, I have had many other coauthors, many of them in physics and in applied science. I learned a lot of ideas and technology from my coauthors. It has been a very rewarding experience.

\section{Question 4}

Qirui Li: Well, my question: when you encounter some hard times while researching, especially when it is hard for you to stick with it, I wonder how do you stick with it? I mean, when you determine to stick to it, what do you do? Do you just think about the problem all day or do you take a rest, such as doing some sport or take a vacation? Because sometimes my teacher tells me that when you encounter a hard time in your research, it is more pleasing to come up with ideas while you take a rest or doing something else. So, I'm very interested in what you do when you encounter such a hard period.

Schmid: Well, I don't think there is a simple answer-it really depends. When one works on a problem, there are different ways of getting stuck. It might be that you are quite certain you will be successful with your problem eventually, that you have merely run into a local difficulty. Then it may be a good idea to do something else for a while: perhaps another mathematical problem, or teaching, can be a good distraction. But what happened to me more than once is that it seemed to me I was really doing something else or thinking about other matters, but somehow, deep in my subconscious, the problem bothered me. And, in the end, somehow the solution came to me when I was unaware that I was thinking about the problem at all. However, I can't say it always happens. One must be honest with oneself: in some situations, one has to decide that a problem is not going anywhere and you must do something else. That is very hard to face. What is crucial is the ability to tell the difference between a temporary difficulty and a difficulty that just cannot be overcome, no matter how hard you try. Being a successful mathematician involves various judgments, and this is one of them. Another one is what problem to choose to work on. I do believe that mathematical success lies in how well one deals with issues of this sort.

Smirnov: Well, the mind works in a mysterious way. I think it is rarely fruitful to work 16 hours a day with a problem for a long time. If you don't get new ideas, you should take a rest. But even then your mind keeps working. Once I was so exhausted with having no progress on a problem that I decided that I would take a break for a 
month not think of mathematics at all. But after two weeks, it became very tempting, and I thought-maybe just a quick peek, just five minutes. I opened my notes, and suddenly it all became clear in a few seconds, as if I had a bunch of puzzle pieces and they all jumped to their places. So, it is important once in a while to step back and relax.

Shing-Tung Yau: I am not sure what "crisis" means in research in mathematics, but I know when we work on a problem that is of serious interest, we often fail. Fortunately, in many cases, after a year, two years, five years, or ten years, I managed to overcome the difficulties. I believe that if a problem is interesting and important in the sense that it will give rise to a deep understanding of the subject, it will be worthwhile to keep on pursuing it until it is solved. In many cases, I worked with my collaborators and often after much communication, we solved our problems. Sometimes, I find my own way to solve it. It may even occur while I am giving a lecture. In general, I am pretty confident that I can solve a problem if it is really interesting, despite I may fail to solve it for a long, long time. It is challenging and I do not consider those times of failure to be a crisis.

\section{Question 5}

Meilei Jiang: Great masters like you must have a lot of experience with students who were successful, and not that successful. So I want to hear about some advice from you for students. What will make a student fail in his academic career? Different people may have different ways to succeed, but people may have some common factors that make them fail. What makes them fail and what makes them successful?

Mumford: This is really hard to answer. I think the important thing is whether or not the students really have an internal drive to keep them thinking about different mathematical problems, and that they don't let this go. Also, I think it is really important to have much curiosity. You may have a good teacher or not, but that's not the key thing. Think about it this way: perhaps just look for a puzzle you really want to solve.

Schmid: I should tell you about a particular course that I am teaching at the moment at Harvard. This course is called Math 55. It's a course for the mathematically strongest of our first year students. The major problem is to get the right students to take this course. At one point, shortly after I came to Harvard, it seemed to me that the course was very successful for a few, but on the whole did more damage than good: it discouraged strong students who felt they could not compete with those at the very top, and then they decided to go into a different field. There are well-known people who fall in this category: a Nobel Prize winner in economics, for example, and one of the best known constitutional lawyers in United States. Some colleagues and I decided to downgrade the course, to call it Math 25, and to teach it at a less demanding level. The idea was to make it less likely for perfectly capable students to be needlessly discouraged by the top performers. After a few years, some of us regretted that Math 55 no longer existed. We decided to have two courses, Math 55 and Math 25. We taught both courses together for the first three weeks, with the two professors taking turns. The one who taught 55 would be more demanding, and the one who taught 25 would be gentler. At the end of the three weeks we gave a little exam and looked at how students had done on the homework problems. Based on that information, we invited some to go to 55 , and only the students who were invited would be allowed in 55. But, after doing this for a few years, the students said they wanted to make the choice themselves. The way it works now is that students sign up for 55 if they want, and we tell them that for the first three weeks it will be relatively easy to switch to 25 instead. This year when I started teaching 55 , on the first day there were over 50 students in the class, for the second lecture it was down to 40 , and after three weeks down to 20-still too many. I find it difficult to convince some students they should not be taking this course. One thing I tell them, entirely truthfully, is something all of us should keep in mind even though it may be difficult to accept: if you look at mathematicians, some are what might be called "fast", meaning that when they talk to other mathematicians, they immediately understand what is being said; there are other mathematicians who are what might be called "slow", in the sense that it takes longer for them to understand. But in the end, a slow mathematician can prove results that as just good, or even stronger, than a fast mathematician. A difficulty in teaching 55 is to convince some students that they will be better off being in the slower course. But that does not mean they cannot be successful. That is something that all of us have to keep in mind.

Smirnov: So, were there any examples of successful mathematicians who used to be expelled from 55? Not successful lawyers, but successful mathematicians?

Schmid: Oh, surely yes.

Smirnov: Well, then maybe it is hard to give good advice. I would suggest reading an interesting book edited by Timothy Gowers a few years ago: The Princeton Companion to Mathematics. It is a very thick book, about 700 pages long, with the ambition to give a snapshot of modern mathematics. One of the chapters, freely available on the internet, has advice from a few famous mathematicians to prospective graduate students. It is a very interesting read, as the advice given is very different-perhaps, there is no single recipe.

Shing-Tung Yau: Over the past forty years, I have had many Ph.D. students. I observed that the best students are those who have strong curiosity and who are hard working. In general, they are solid in their basic 
knowledge and also curious about new subjects that they do not know well. In most cases, one needs to train yourself during the time that you want to solve a problem, and you need to learn the technology by consulting with people and by reading. Once the basic training is done well, strong curiosity will lead you to a discovery of importance.

\section{Question 6}

Jinbo Ren: Okay, my question is especially for $\mathrm{Mr}$. Mumford. My question is about learning algebraic geometry, which is my main interest. I am just a beginner and I have heard, you know, many of my friends, for example, say that they tried to learn by following the example, and the language, of Grothendieck. You know, they tried to read Hartshorne's book. Only a minority of them can understand all of it, the majority of them, after reading one third of the book, said, "oh, what the hell! What does it mean?" And so they gave up. They said, "all I know is a few definitions and some theorems. I can remember all of the details of the proof, but I know nothing about the language of Grothendieck!" I know it is very important, but it is not easy for me to learn. So what's the reason for their failure, and what's the best method to understand the language and thoughts of Grothendieck. Thanks.

Mumford: Well, I think there are really two different approaches to learning a subject. Probably three quarters are in one category and one quarter in the other category. The three quarters is that they really have to learn something by starting with concrete examples, and working their way up, through different levels of generalizations and abstractions. One quarter of people, well, they work the other way around and for them, this makes things much clearer. They start with most abstract point of view and they can specialize from that. This seems to me the more difficult approach, so I would say that to start with Grothendieck is not a good idea for most people. I mean, really, Grothendieck's book is basically just a language. What you really need to do is to get to the substance of algebraic geometry: questions like desingularization, linear systems, and cohomology. Another way is to start more historically, with curves, genus, and Riemann-Roch. Then when you have the basic stuff under your belt, and you begin to see the great advantage of this language that Grothendieck created. But I personally wouldn't want to start with something like that.

Schmid: Let me tell you about my own experience in graduate school. After a year and half as a graduate student, my PhD thesis advisor told me it was time to work on a thesis. He gave me three problems to choose from. That did not mean I couldn't choose my own, but they were good suggestions. I ended up picking one that involved Lie theory, a subject I had not studied before, and I found that by looking at this concrete problem, I learned the subject much faster than I would have any other way.
By focusing on one particular problem, one can often learn a new subject far more efficiently than in lectures.

Smirnov: I think I perfectly agree. It is much easier to learn something if you have a specific problem in mind. I have friends who can easily read and digest a book without any particular need, but this never worked for me. Besides, most textbooks are written without describing the history or intuition behind the subject. I remember reading the famous book of Herbert Federer about geometric measure theory as an undergraduate.

\section{Mumford: Oh my god!}

Smirnov: Yeah, exactly. And then I stumbled upon Frank Morgan's book which, in much a more intuitive way, introduced the main ideas. There were many pictures in this book explaining why these things are needed. It doesn't do the difficult proofs, but if you read it, you get some intuition. It also helped understand what exactly I needed from Federer's volume and I managed to read it, and use it several times. So, besides having particular problems in mind it is good to start with some more accessible texts.

Shing-Tung Yau: I like to concentrate on concrete problems, either in geometry or in physics. Only with such problems, am I excited. When I am excited by a problem, I try to find good ideas to solve the problem, either by myself or with friends. Sometimes, we have such good luck that we can solve the problem quickly. But often it takes a long time, even ten years. However, I was confident in solving problems that I was excited about. When I solved a concrete problem, I felt that I got something solid. Hence, I stick to concrete problems. Some books are tough to read: like the book of Federer on geometric measure theory. When I was a first year graduate student, I had a course on partial differential equations, by Charles Morrey, at Berkeley. He had just finished writing a book called Multiple Integrals in the Calculus of Variations. He did not quite use that in his class, but he taught materials related to that, and eventually I was the only student in his class. I read his book and it was tough, but I persisted. He wrote it in a way that was difficult to follow. Often, when we read the first few chapters, we were referred to materials in the later chapters. I think many analysts refuse to read this book-there was an easier book written by David Gilbarg and Neil S. Trudinger. It is well written and I promoted this book to my friends and students. But the content of the latter book is not quite the same as in Morrey's book. Morrey is the master in the subject. One can see his original ideas despite the book being difficult to read. It is the same with the book by Federer. By reading books and papers of masters of the subject, one sees global ideas. Even if it is tough to read, we should still try.

\section{Question 7}

Audience Member: I have a question I want to ask 
Prof. Smirnov. I attended your speech about discrete complex analysis and planar statistical models. These are different from each other-not mathematical neighbors. How did you find the path to link these domains? I suppose there four possibilities: the first is that you researched discrete complex analysis first, and then you wanted to find some applications, and found that it can be applied to planar statistical models. The second possibility is that you researched the models first, and then you wanted to find tools to work with in these models, and it turned out that discrete complex analysis could provide them. The third case would be that you learned each branch, and so you knew these different domains could be bridged naturally. Lastly, you knew about the mathematics from your speech. The fourth case would mean you are a genius, and you are capable of knowing that they are linked merely by your strong intuition. So which one is the case?

Smirnov: Well, I think the question was about what the best way to do mathematics is. You can start from a problem and then try to find or create a tool to solve it. Or, you can master some tools and then look for interesting problems where they can be applied. I certainly know people who have proved important theorems following successfully either of these approaches. So you should decide for yourself.

I personally prefer the first approach, much as Lennart Carleson who was my mentor. You choose some problem which you find exciting, and where you have a couple of ideas of how to start, and then you try to find the tools, or to create them. But it can take a long time, and indeed, as Richard Feynman used to say, it is better to have "a few trumps up your sleeve" before you start-some relevant ideas other people have not tried before.

The percolation problem I spoke about has an interesting history in this respect. It appeared in one of the first volumes of "American Mathematical Monthly" in 1891, as a problem for schoolchildren, as it is quite easy to formulate: in a box, which is randomly stuffed with white and black balls, what is the probability that there is a path from one side to another? A "solution" was even published, but it addressed an oversimplified case looking for a straight path, and even then it was wrong! The editor felt that something is missing, so there was a footnote saying that a complete solution would be welcome. It was in 1891, and then somehow the problem died out as there was no motivation, besides pure curiosity. It resurfaced in the 1957 paper by Broadbent and Hammersley, who were motivated by physical processes of gas or liquid percolating through a porous medium. Much activity was generated, but most questions seemed unanswerable until intuition from other areas of physics-renormalization theory, and then conformal field theory-became available, and led to several spectacular conjectures, like percolation cluster being almost surely of dimension 91/48. Still, this seemed out of reach mathematically, since physics arguments were hard to formulate precisely. Many people were contributing, bringing different pieces, and it took some twenty years to have a fairly complete picture. Interestingly, though the progress was motivated by physics, the resulting arguments are different, and are of interest to physicists themselves.

Well, I don't know I answered you question. So, what is your opinion, Wilfried? What is the best thing to do: start with a tool and look for problems or start with a problem and look for tools?

Schmid: Certainly the latter. The people who first acquire a method and then look for a problem with that method are perhaps somewhat restricted. The problem should be really interesting and you should find tools for it. You build a house, and then you choose tools necessary to do that. You don't get tools and then build the house you can build with these tools. Of course, if the problem is too far from your area of expertise, then it may be unattainable.

Mumford: Let me just say something my Ph.D. advisor Oscar Zariski said, which stuck with me. He said that it is a terrific thing to prove a new theorem, but it's really important to derive a new technique. That is really true. I think I was tremendously fortunate that I came along when Serre had made the first use of cohomology, showing that algebraic geometry had cohomology hidden under the surface. This was waiting to happen and I was the lucky one who grasped the power of his ideas. I think inventing a new tool is more important often than building a new theory.

Shing-Tung Yau: As I said, my motivation in mathematics is to understand the true nature of geometry and it has to be understood through concrete problems. In order to do that, I need to develop tools. I do not work on mathematics because I have learned some tools and want to find a problem to be solved by these tools. On the other hand, when the tools that I developed seemed to be powerful for solving other problems, I paid attention to them. For example, about thirty years ago, Peter Li and I developed some basic estimates for parabolic equations. I felt it can be applied to other concrete parabolic equations.

When I met Hamilton in San Diego, I told him that it is important to find the analogue of such an estimate for his Ricci flow. He was convinced by me that it is important and after a few years of work, he found the right generalization of Li-Yau estimate for Ricci flow. That became fundamental to all later work on Ricci flow in understanding singularities of the flow.

So, in this case, we found an important tool and applied it to some important problem. But the tool was new and the problem was important and natural. Therefore, in such a situation, we can find a problem to fit a tool. In many cases, however, people worked on boring problems because they have just mastered some specific tools and they wanted to find problems that the tools can be ap- 
plied. That is not what I like to see.

\section{Question 8}

Fuzhou Wu: I have a question for Prof. Yau. Is it true that mathematicians are studying the framework of topological string theory, while physicists study that of ordinary string theory (e.g. Type I string theory, Type II string theory)? Suppose that the LHC cannot find the Higgs particle after all-it would probably mean that the string theory is at risk. However, even if string theory is dead, the mathematics still remains unaffected. When you investigate string theory or string geometry, are you worried that your theory will never be verified by evidence from a particle collider, or from astrophysical observations like black holes?

Shing-Tung Yau: I think you are asking me about the mathematics motivated by string theory. This area of mathematics is fine, whether or not string theory can be tested to be correct. Many of the statements motivated by string theory can be proved with a rigorous mathematical treatment. An example is the mirror principle that Bong Lian, Kefeng Liu, and I proved, which was also found independently by Givental. It should be interesting to know that, while we can prove the formula, it was difficult to find the statement without knowing the motivating material coming from the physics of string theory. Hence, they do have good intuition and often the statements motivated by them are spectacular and powerful. String theorists do not make many mistakes in their predictions of statements in mathematics. Therefore, there must be some truth in the reality of these ideas.

\section{Question 9}

Another Audience Member: As a supervisor, I want to know in what ways you can guide students into the right direction. Since students are very different, what do you think is most important for guiding he or she toward the right direction, in order to find a fulfilling career, or research, or something like that?

Lizhen Ji: Any volunteers?

Smirnov: Who is the most experienced?

Mumford: Everyone is different.

Smirnov: Like every child.

Lizhen Ji: Maybe I can change the problem into one like this: some of you have talked about leaning from books, and I think leaning from people is also important. So, what is the best way to learn from people? For example, this conference is called Master Lectures on Mathematics: what is the best way to learn from the masters?

Mumford: I can't answer your question in any way at all. But there is something I find remarkable. I have had the experience a half dozen times, at least, where I have gone to a lecture, listened, and found it was interesting. Afterwards I thought I had forgotten all about it. But sometime later something else happened and I had what I thought was a new idea until I realized, "my god! That is the idea that person had talked about, which has somehow come back into my mind!" So, you never know what you can get from people. It's a very strange process.

Schmid: I have a question for you.

Lizhen Ji: Yes, for me?

Schmid: I didn't recognize her original question in your paraphrase at all.

Lizhen Ji: She asked how to direct a student toward the right direction. In my reformulation, what's your advice to take advantage of talking to a master?

Schmid: As far as the original question is concerned, I think all three of us have exactly the same reaction, namely that every student is different. What works for one student may not work for another. To give a definite, clear answer is almost impossible. But I should also say that some of us are better and some are worse at advising students; this is not something mathematicians are trained for. We have to, as one says in English, fly by the seat of one's pants.

Shing-Tung Yau: I think students should learn from both books and lectures. Many students refuse to go to listen to lectures which seem to be irrelevant to their own field. This is not healthy. I learn a lot by going to lectures that seem too far from my interests. In fact, my thesis is related to infinite group theory, which I learned about through a conversation with my college professor, but I did not take any courses on the subject. I remember that one of the most wonderful experiences I had was to listen to the lecture given by David Mumford in UC Irvine in 1976, when I just finished my proof of the Calabi conjecture. David was giving a lecture on algebraic geometry at UC Irvine, which is quite far from my home. It took about a two and half hour drive for me. I drove all the way just to listen to his talk. I learned from David some open problems in algebraic geometry that I found exciting. Then I remembered what I did a couple of years ago when I tried to give counterexamples to the Calabi conjecture. I did those calculations before and they fit well with the open problems mentioned by David. By the time I arrived home, I realized that I could solve those open problems. It was truly exciting! I was really fortunate to listen to a lecture that was not in differential geometry. David did not really know me at that time.

Mumford: I was thinking: who is this young guy? It seems that he claims that he can prove all this stuff.

\section{Question 10}

Lizhen Ji: More questions for them? I think that you have asked a question before. 
Jiayin Guo: When I am learning, I always face two types of problems. One of these problems is based on knowledge I know much more about. And another type of problem involves many areas of knowledge. And there are some areas I simply know little of. I want to speak more about the former problem. To solve the former, I use some isolated methods and some basic knowledge. So, my question is for these two types of problems, which one should I spend more time on?

Schmid: If I understand you correctly, you are asking about two types of problems: one, about which you think you know all the tools necessary to solve it; the other, which you think requires tools you have not mastered yet. My answer is that it should be a problem that really excites you! As I said before, picking the right kind of problem to work on is an important part of becoming a mathematician.

Mumford: You have a problem, and you get the sense that something is going on, which isn't clear. Somehow you have the sense to dig deeper in this direction and you get sucked in.

Smirnov: I think I totally agree that you should look for problems which interest you more, rather than whether you have all the tools.

Shing-Tung Yau: Yeah, I agree with that. When you are excited by a problem, you will feel that it is so important that you need to develop all tools to solve it. I was excited by the Calabi conjecture because it provides a basic understanding of what Ricci curvature of a Kähler manifold is. I felt that it was extremely important as it will open the gates to understanding Kähler geometry. So I developed tools and all the necessary analysis to overcome the difficulties. I think the same feeling happened to my friend Hamilton when he developed Ricci flow. There were many, many difficult obstacles he needed to overcome. He was so excited by the outcome that he solved these obstacles one by one over twenty years.

Hence, when you are excited by a problem, you will have strong motivation to develop the methods to solve it-even though such tools may be tough to develop.

\section{Question 11}

\section{Lizhen Ji: Maybe let's have one more question.}

Cheng Jing Rui: I want to ask how one should decide what to learn. Should we only learn those portions of mathematics which are necessary in one's research?

Mumford: Try to learn a bit of everything. You really should try to pick up as many tools as you can when you are a student. Things cross fertilize each other.

Schmid: Maybe I slightly disagree with that. It makes no sense to become a mathematician, to work in mathematics, unless you have already a pretty good grounding and good basic knowledge in different areas of mathe- matics. But I would still say that if you have a problem that excites you, then you take that problem and you'd better acquire those pieces of mathematics that you need to solve it.

Smirnov: I would say that it's good to have some all-around basic knowledge so that when it comes to you that you have to use certain methods, you would understand it and know where to read or whom to ask. Most likely you won't need to have concrete knowledge of algebraic topology if you work in harmonic analysis. But you never know, and you should be able to recognize the need if it ever arises.

Mumford: Modular forms came up in algebraic topology. Who had thought that would happen? Nobody!

Smirnov: Here I think one should mention that probably you have more time to read books as a graduate student or as a post doc than later in your life. These are the few years that you have much more time to read books, especially outside your area, compared to later. And later you'll have some time to read books in your area, but you're unlikely to have the luxury of reading many outside.

Mumford: I regret a lot that I didn't take any course in probability in graduate school and it was such a struggle to learn it later.

Shing-Tung Yau: When you are young, you should learn as much as possible, especially when you are an undergraduate or graduate student. You actually have much more strength to learn compared to later days when you are very busy. When you are in graduate school, you should learn at least 2 major technologies in different fields that you feel comfortable with so that you can cross disciplines in order to understand each subject when you need to. I regret that I did not learn quantum physics, or quantum field theory well, when I was in graduate school or in college. When you are a graduate student, you are expected to learn. But after you graduate, you become a faculty member in some university where you are expected to be expert in that subject immediately. And by that time, you may fear to get into a new field. Also you will have much less time to learn some new subjects.

Getting to know a new direction is very important when you are in graduate school. This is the time when you have time to learn more technology. And also, when you are young, you have much bigger capacity to learn than when you are getting older. When time goes on, I found myself learning much less, for many different reasons. When I was a graduate student, I did not understand most mathematics, but I studied most of the papers in every journal. I was sitting in the Berkeley library, reading almost every journal, although I did not understand most of them, but some of these have become very useful for me. Unfortunately, now I can not do it-I'm far from being able to do it. Maybe I am getting much older... 
Smirnov: There are also more papers! There are 90,000 per year on MathSciNet, and it is too much for anyone...

Shing-Tung Yau: Yeah, it's true. Forty years ago, there were much fewer papers, and I could read most of them. Although I did not understand most of them, some of them have been very useful for me. I think that when you are a graduate student, you should just learn as much as possible. Try to master at least two technologies, which may be very useful for you. Master them --don't just know them-which means that you should be able to use the technology to solve problems.

\section{Question 12}

Lizhen Ji: Let me combine that set of questions into the last one. Today is an interaction between the West and the East. We have the best people from the West and the best students from the East. I think most of them will go to the West to study. Can you comment on the difference between the West and East and, if they go abroad, what is your best advice for them?

Schmid: I think you can certainly become successful studying mathematics in China. But my own experience goes back much further, to when I was a graduate student. At that time, it wasn't thought that you needed to go somewhere else as a student. But once you had finished your $\mathrm{PhD}$, it was considered important to get some experience in other countries. I think what is important is to see mathematics not in just one university, or two universities, but to see what is going on elsewhere. And whether this happens when you are a student, or afterwards, maybe that does not matter so much.

Smirnov: With experience, I think it is really important. There are some European countries, such as Sweden or Austria, where you cannot get a permanent position unless you have spent a couple of years abroad. It's a very good rule and it works really well. As for choosing places for PhD study, I think at this stage the main thing is to choose an advisor, which is different from that of an undergraduate. It's better to have a good idea before starting to work with someone, say meeting this person at a summer school. That is more common in Europe-to come to graduate school having a specific advisor in mind.

Schmid: I would like to add that, typically, in the United States you apply to a department and you don't choose an adviser until you are in that university. You know who teaches at which university but you don't apply to Yau to become a graduate student. You apply to the department of mathematics at Harvard. But I believe that there are certain European countries where, in fact, it doesn't work exactly the same.

Smirnov: No, you usually apply to the department, but often you work with a certain professor whom you have met before and that works the best.

Schmid: Yes, but that's not typically how it works in the United States.

Mumford: Unfortunately, finding the perfect advisor accidentally is rare. It's hard. I don't know the situation in China, so I don't know whether it's better to do your graduate training here. One thing that is certain in the US is that if you are in large place, such as the Boston area, you will hear so many people coming through, so you get an impression of many different professors and their personalities.

Smirnov: That could even be a problem if you are in New York or Boston. There are so many people passing through, there are so many interesting seminars, and you have so few hours left to work. That's maybe too distracting for a graduate student, even for a professor. And it could be difficult to choose an advisor when there are too many choices. So I don't know... perhaps, indeed, the best idea is to go to a couple of summer schools when you are an undergraduate, trying to meet people. At least in Europe there are now many possibilities, and also there is a high mobility of undergraduate students. Many of them go on exchange trips. But I don't know how the Chinese system works now.

Shing-Tung Yau: In China, the system of higher education has been changing, and I hope changing for the better. The Chinese universities, in general, want to keep their own students and their own faculty in their department. This has been tough on students and also to the faculty who want to move around to learn about new cultures from other department. You probably don't know much about the system in China. Let me give an example: if Tsinghua University wants to hire a faculty member from Fudan, Fudan can just keep him in Fudan-not allowing him to go to Tsinghua-despite the faculty member wanting to go himself. So, in the end, you might end up with him having two positions, which I think is very unhealthy. Most universities also want to keep their own graduate students who are good. This is rather unusual compared to the American system where they always encourage their graduate students to go to work in other universities when they graduate. American universities also encourage their own undergraduate students to be graduate students in other places.

This is not quite possible in China; however it's changing, and I hope changing for the better. In the other direction, I think that Chinese students need to be more open-minded when they come to America. They should learn more subjects that they have never studied before. The situation in China has improved a great deal.

Ten years ago, a student could probably learn very little in China, but now I feel that there are many capable professors coming to China. In some major universities, there are as many capable professors as in America universities. You can encounter many first class mathemati- 
cians more often than many places in America. Harvard is a great university, but we don't have as many faculty members working at Harvard in the summer. You can find more people in Beijing during the summer. It's useful for graduate students to go to Beijing to learn from these faculty members. I am not sure how active Shanghai is, but Beijing is active and you can learn a lot. By learning, I mean you obtain serious knowledge. You learn how to do calculations, you get global ideas, and many other interesting things. But make sure that you have actually learned something, not just sat there listening. I find many Chinese students are not taking notes nowadays, compared to the old days. Many of them just sit there, and don't take notes. They will not have much reference to learn later. I think you should learn with serious attitude, and you will be much better off in that way. But, as I said, the Chinese system is getting closer and closer to the Western system. I hope that in five years, they will be mature enough to produce large number of first class students within China.

Lizhen Ji: So let's thank all of the masters for sharing their experience and giving valuable advice.

\section{Acknowledgements}

I would like to thank Youjin Zhang for organizing students at Tsinghua University for transcription of these conversations, and the students-Qirui Li, Chenglong Yu, Wei Chen, Jingrui Cheng, Meilei Jiang, Jiayin Guo, Jinbo Ren, Ziliang Chen, Huachen Song, et al-for carrying out this challenging project. 